\title{
química
}

\section{AVALIAR A COMPOSIÇÃO QUÍMICA DO MESOCARPO DE BABAÇU (Orbignya oleifera) in natura NO MUNICÍPIO RAPOSA-MA}

Kiara Janda Ferreira Sudre ${ }^{1}$, Angela Maria Correa Mouzinho Santos², Lucy Rose de Maria Oliveira Moreira ${ }^{3}$.

1,3 Instituto Florence de Ensino Superior, IFES, São Luís - Maranhão - Brasil.

2 Instituto Federal do Maranhão, IFMA, Santa Inês - Maranhão - Brasil.

Resumo: O babaçu é uma planta nativa do Brasil, disseminada por quase todo o interior do país, desde o estado do Amazonas até o estado de São Paulo. O babaçu (Orbignya oleifera) é uma palmeira amplamente distribuída no Maranhão onde o mesocarpo é usado na medicina popular e como suplemento alimentar, e tem, hoje, a possibilidade de ser aproveitado também na área farmacêutica. Tendo em vista essas diversas potencialidades, busca-se, atualmente, o desenvolvimento tecnológico de novos produtos a partir do mesocarpo do babaçu, para isto, determinados estudos preliminares assumem grande importância. Diante do que foram expostos ressaltando a importância do seu uso popular, características físico-químicas constatadas em várias plantas do gênero Orbignya. Sendo assim o presente trabalho teve como objetivo geral avaliar a composição química do mesocarpo do babaçu (Orbignya oleifera) por meio da Espectrometria de Emissão Óptica com plasma indutivamente acoplado - (ICP OES), observou-se a presença do potássio (K) com 10,15 $\mathrm{mg} / \mathrm{L}$, cálcio $(\mathrm{Ca})$ 9,22 mg/L, magnésio $(\mathrm{Mg})$ com 3,56 mg/L, fósforo $(\mathrm{P})$ com 1,56 mg/L , mercúrio $(\mathrm{Hg})$ com 1,41 mg/L e iodo (I) com 1,27 mg/L respectivamente. Enquanto na análise na região do infravermelho observou-se bandas de intensidade fraca e muito forte, respectivamente, devido às vibrações de estiramentos assimétricos acoplados à ligação de cadeia longa da função éster.

Palavras-chave: Orbignya oleifera, mesocarpo, babaçu, composição, química.

\section{ASSESSING THE CHEMICAL COMPOSITION MESOCARP BABAÇU (Orbignya oleifera) in natura IN THE CITY RAPOSA-MA}

\begin{abstract}
Babaçu is a plant native to Brazil, spread over almost the entire interior of the country, since the state of Amazonas to the São Paulo state. The babaçu (Orbignya oleifera) is a palm tree widely distributed in the Maranhão where the mesocarp is used in folk medicine and as a food supplement, and today has the possibility of being taken advantage also in the pharmaceutical area. Given these various capabilities, we seek today the technological development of new products from the babaçu mesocarp to this, certain preliminary studies are very important. Before they were exposed, highlighting the importance of its popular use, physico- chemical characteristics found in various plants of the genus Orbignya. Thus the present study was to evaluate the overall chemical composition of the babaçu mesocarp (Orbignya phalerata Mart) by Optical Emission Spectrometry with Inductively Coupled Plasma - ( ICP OES ), the presence of potassium (K) was observed to $10.15 \mathrm{mg} / \mathrm{L}$ calcium $(\mathrm{Ca}) 9.22 \mathrm{mg} / \mathrm{L}$, magnesium $(\mathrm{Mg}$ ) with $3.56 \mathrm{mg} / \mathrm{L}$, phosphorus ( P) with $1.56 \mathrm{mg} / \mathrm{L}$, mercury ( $\mathrm{Hg}$ ) with $1.41 \mathrm{mg} / \mathrm{L}$ and iodine ( I) with $1.27 \mathrm{mg} / \mathrm{L}$ respectively. While the analysis in the infrared region was observed bands and very strong weak intensity, respectively, due to the asymmetric stretching vibrations coupled to the connection of long chain esters function.
\end{abstract}

Keywords: Orbignya oleifera, mesocarp, babaçu, composition, chemistry. 


\section{INTRODUÇÃO}

A região em que os babaçuais apresentam expressão significativa representa aproximadamente a quinta parte do território do Maranhão. O babaçu é uma planta de pleno sol, com um comportamento de invasora, após os desmatamentos de matas naturais. As matas da região ao longo do rio Itapecuru foram desmatadas no século XVIII, servindo as terras para a produção de algodão. No final do século os babaçuais começaram a ser explorados pelo óleo, para atender o mercado nacional e após a segunda guerra mundial, passaram a suprir a demanda internacional [1].

O babaçu é uma planta nativa do Brasil, disseminada por quase todo o interior do país, desde o estado do Amazonas até o estado de São Paulo. Segundo Lorenzi [2], é no estado do Mato Grosso, na região Norte e parte da região Nordeste do território brasileiro (nos estados do Maranhão, Piauí, e algumas áreas isoladas no Ceará, Pernambuco e Alagoas), onde se localizam as principais ocorrências dessa palmeira. A ocorrência do babaçu em diversos Estados brasileiros é justificada pela tolerância a climas com temperaturas elevadas e constantes e precipitações pluviométricas acima de $1.000 \mathrm{~mm}$ anuais, condições verificadas, especialmente, nas regiões de cerrado, cocais e baixadas [3].

A palmeira de babaçu (Orbignya oleifera) tem aproximadamente 15 metros de altura e costuma produzir mais de 500 frutos a cada florada [4]. Dessa palmeira podem ser extraídos cerca de 68 sub-produtos. A palmeira de babaçu possui três estágios de crescimento. O primeiro, constituído pelas pindovas, é quando a palmeira apresenta até três folhas definitivas [5]. O segundo, denominado palmiteiro, pode ser identificado pelo palmito, quase ao nível do solo. No terceiro, o caule já se encontra formado, correspondendo à fase anterior à fase adulta [6,7].

Segundo Lorenzi [2] a planta do babaçu apresenta caule solitário, colunar, de 10-30 m de altura e 30$60 \mathrm{~cm}$ de diâmetro. Suas folhas são pinadas, eretas e divergentes, com 175-260 pinas regularmente distribuídas sobre toda extensão da raque [8], as flores, geralmente, são creme-amareladas, estaminadas [9], distribuídas em duas fileiras ao longo de um dos lados das raquilas [10]. Segundo Wilhelms [10], a palmeira do babaçu "floresce de novembro até janeiro, sendo que o período de desenvolvimento dos cachos que aparece nas inflorescências [12] femininas vai de julho até novembro". Ressalta, ainda, que cada palmeira produz anualmente dois, três e até quatro cachos pêndulos de um a dois metros de comprimento.

O babaçu é constituído de quatro partes principais: o epicarpo, o mesocarpo, o endocarpo e o fruto (amêndoa) [6,7]. A amêndoa do babaçu pode ser extraída de diversas formas, mas em geral, a sua retirada é feita com o auxílio de um machado e de um pedaço de madeira (porrete) [8]. Para quebrar os cocos, os trabalhadores se sentam prendendo o cabo do machado abaixo de uma de suas pernas e seguram o coco inteiro com uma das mãos acima da lâmina do machado para desferirem o golpe de porrete sobre o coco com a outra mão [9].

O babaçu (Orbignya oleifera) tem sido um recurso natural importante para o Maranhão, seja pela preponderância que assumiu no âmbito comercial, seja pela sua relevância junto às famílias rurais do estado [9]. Nessa perspectiva, faz-se um breve panorama da inserção do babaçu nos circuitos de mercado, quando esse produto passou a ser considerado importante para a economia maranhense [9]. 
O babaçu (Orbignya oleifera) é uma palmeira amplamente distribuída no Maranhão onde o mesocarpo é usado na medicina popular e como suplemento alimentar, e tem, hoje, a possibilidade de ser aproveitado também na área farmacêutica. Tendo em vista essas diversas potencialidades, busca-se, atualmente, o desenvolvimento tecnológico de novos produtos a partir do mesocarpo do babaçu, para isto, determinados estudos preliminares assumem grande importância. Do mesocarpo é obtida uma farinha amplamente comercializada e utilizada pela população como alimento e como medicamento. Dados etnobotânicos indicam que essa farinha pode ser utilizada no tratamento de feridas crônicas, úlceras gástricas, úlceras duodenais, inflamações diversas, tumores, obesidade entre outras doenças [10].

Segundo relatos populares, o mesocarpo de babaçu apresenta algumas propriedades medicinais. Sousa afirma que as mulheres quebradeiras de coco utilizam o mesocarpo de babaçu no tratamento de gastrites e doenças inflamatórias. Acredita-se, também, que o mesocarpo pode trazer benefícios para dores, constipação, obesidade, leucemia, dores menstruais e entre outras doenças. Vários trabalhos vêm demonstrando o interesse de estudar as possíveis propriedades medicinais do mesocarpo, tendo como exemplo o trabalho de Azevedo e colaboradores que comprovou o beneficio do mesocarpo como tratamento para trombose, a partir de um estudo in vivo com camundongos [11].

Diante do que foram exposto, ressaltando a importância do seu uso popular, características físicoquímicas constatadas em várias plantas do gênero Orbignya, o presente trabalho teve como objetivo geral avaliar a composição química do mesocarpo do babaçu (Orbignya oleifera) por meio da Espectrometria de Emissão Óptica com plasma indutivamente acoplado - (ICP OES) e análises na região do infravermelho obtido na Cidade de Raposa - MA.

\section{MATERIAL E MÉTODOS}

\section{Coleta do mesocarpo do babaçu Orbignya oleifera}

Realizou-se um estudo analítico qualitativo e quantitativo do mesocarpo do babaçu (Orbignya oleifera). O material vegetal foi coletado em julho de 2013, na cidade de Raposa, Estado do Maranhão, Brasil. Os frutos coletados de Orbignya oleifera foram secos a sombra e extraídos mecanicamente por meio de peneiras com furos de diferentes diâmetros $(25 ; 31,5 ; 37,5 \mathrm{~mm} /$ microm). Após o processo de separação do mesocarpo, procede-se a moagem do mesocarpo para a obtenção do pó do babaçu. A exsicata ${ }^{\circ} 01022$ da espécie Orbignya oleifera, encontra-se depositada no Herbário Ático Sebra da Universidade Federal do Maranhão (UFMA).

\section{Determinações de metais em minerais no mesocarpo de babaçu Orbignya oleifera (ICP OES)}

Para este teste, foi pesado $0,25 \mathrm{~g}$ do mesocarpo do babaçu e acrescentou-se $3 \mathrm{~mL}$ da solução de $\mathrm{HNO}_{3}$ e colocou-se no sistema de decomposição pressurizada com aquecimento convencional Berghof (bomba de digestão). Logo em seguida colocou-se a amostra na estufa a uma temperatura de $180{ }^{\circ} \mathrm{C}$ por um 
período de uma hora e trinta minutos $(1: 30 \mathrm{~h})$ para que ocorresse a digestão. Após esse período a amostra foi armazenada em um balão volumétrico de $25 \mathrm{ml}$ com água deionizada para a volumagem.

\section{Caracterizações dos compostos químicos por Espectrometria de Emissão Óptica com plasma indutivamente acoplado - (ICP OES)}

As análises para identificação de metais foram realizadas na Universidade Estadual do Maranhão (UEMA) no Laboratório de Solo, utilizando um Espectrômetro de Emissão Óptica com Plasma Indutivamente Acoplado modelo Vista-pró, simultâneo e com configuração axial (Varian 720-ES). A técnica de ICP- OES utiliza o plasma como fonte de excitação. O plasma normalmente é formado pela ionização parcial de um gás, geralmente o argônio, em um campo magnético oscilante produzido e mantido por uma fonte de radio frequência. Este estado parcial de ionização, induzido pelo campo magnético, ocasiona um aquecimento ôhmico proporcionando temperaturas de até $1000^{\circ} \mathrm{C}$. A alta temperatura e a atmosfera inerte do argônio no plasma minimizam as interferências não espectrais, melhorando a sensibilidade, precisão e a exatidão da técnica.

\section{Análises no infravermelho}

A espectrometria é o processo analítico-instrumental baseado nas propriedades de absorção, emissão e reflexão de energia eletromagnética em região específica do espectro [12].

\section{Preparo das pastilhas de $\mathrm{KBr}$}

$\mathrm{O} \mathrm{KBr}$ foi previamente dessecado em estufa à $120^{\circ} \mathrm{C}$ até peso constante e triturado em almofariz . Posteriormente foram colocados $0,1 \mathrm{~g}$ do $\mathrm{KBr}$ no pastilhador seguido de compressão em prensa hidráulica Modelo GrasebySpecac com pressão de 15 toneladas, para obtenção das pastilhas finas e transparentes, a fim de garantir que a pastilha seja translúcida pela qual a luz pode passar. Portanto, o brometo de potássio não absorve radiação infravermelha, então as únicas linhas espectrais a aparecer serão da amostra.

\section{Analise em pastilhas de $\mathrm{KBr}$}

Colocou-se a amostra do mesocarpo do babaçu (Orbignya oleifera), sobre as pastilhas de $\mathrm{KBr}$ com auxílio de capilares de vidro. As pastilhas obtidas foram inseridas no compartimento de leitura do espectrofotômetro do infravermelho marca BOMEM, modelo MB - Series e analisadas, obtendo-se os espectros da composição química do mesocarpo de babaçu (Orbignya oleifera) faixa de $4000-400 \mathrm{~cm}^{-1} \mathrm{com}$ resolução de $4 \mathrm{~cm}^{-1}$.

\section{RESULTADO E DISCUSSÃO}

A ocorrência da planta do babaçu em diversos Estados brasileiros é um quesito importante, quando se busca saídas, ou seja, alternativas de desenvolvimento local a determinados territórios. As potencialidades do babaçu são inúmeras, da geração de energia ao artesanato, diversas atividades econômicas podem ser desenvolvidas a partir da palmeira. Dentre as partes desta, o fruto tem o maior potencial econômico para aproveitamento tecnológico e industrial. 
Segundo Migliato et al.; Gobbo-Neto \& Lopes [11,13,14], algumas substâncias químicas são características de uma determinada espécie vegetal, servindo como parâmetros para a sua caracterização e identificação.

Segundo Rosenthal, o mesocarpo do babaçu tem em sua composição 68, 3\% de amido e 1,25\% de fibras de aminoácidos. Além disso, apresenta hemicelulose e pentosanas e entre outras substâncias químicas. O mesocarpo do fruto do babaçu, devido a sua composição química, as suas propriedades medicinais e ao seu elevado teor de amido, que segundo Peixoto [8] varia de 63,75\% a 71,29\%, tem possibilitado o seu uso em áreas diversas, como na nutrição humana.

Para determinação de metais e minerais no mesocarpo do babaçu (Orbignya oleifera), usando a técnica de ICP OES. Conforme demonstra na figura 1, observou-se que na amostra analisada não houve muita diferença nos resultados, pois notou-se a presença elevada dos mesmos metais potássio (K) e cálcio (Ca).

Na figura 1, observou-se a presença de potássio (K) com 10,15 mg/L, cálcio (Ca) 9,22 mg/L, magnésio $(\mathrm{Mg})$ com 3,56 mg/L, fósforo $(\mathrm{P})$ com 1,56 mg/L, mercúrio $(\mathrm{Hg})$ com 1,41 mg/L e iodo (I) com $1,27 \mathrm{mg} / \mathrm{L}$. Alguns metais são considerados essenciais ao ser humano como o potássio $(\mathrm{K})$, cálcio $(\mathrm{Ca})$, magnésio $(\mathrm{Mg})$ e fósforo $(\mathrm{P})[16]$.

O potássio (K) é o sétimo elemento mais comum que ocorre na crosta terrestre, estando largamente distribuído, mas devido a sua alta reatividade e afinidade com outros elementos nunca ocorre em sua forma elementar. Ocorre em rochas, solos, oceanos, lagos e salinas residuais de lagos salgados, embora nestas ocorrências raramente seja observado um teor superior a 10\%. Teores mais elevados são observados nos minerais evaporativos e nos silicatos de potássio [17]. O potássio, por exemplo, elemento imprescindível à vida vegetal, encontra-se nos solos sob a forma trocável, em teores muitos baixos [18].

O potássio e um elemento essencial para a vida vegetal e para vida humana, pois no metabolismo das plantas, o potássio e absorvido do solo na forma de tartaratos e de oxalatos que, por sua vez, podem ser convertidos em carbonatos quando as plantas são queimadas, na vida humana os íons de potássio se junta com o íon de sódio em seguida agem nas membranas celulares na transmissão de impulsos eletroquímicos dos nervos e fibras musculares e no balanceamento da atividade de alimentação e remoção de subprodutos, nas células. Contudo, a mera presença de potássio nos solos já garante a necessidade mínima indispensável do mesmo através da nossa alimentação [17,18].

O cálcio $(\mathrm{Ca})$ é o quinto elemento mais abundante da Terra. Ele corresponde a cerca de $3 \%$ da massa da crosta terrestre e a cerca de $8 \%$ da crosta da Lua. O cálcio vem sendo bastante estudados nos últimos anos por vários pesquisadores principalmente para aplicações tecnológicas na área de saúde voltada ao uso clínico, com o intuito de substituir o enxerto ósseo autógeno em cirurgias ortopédicas [19].

O magnésio $(\mathrm{Mg})$ é de suma importância no uso do dia a dia, todo mundo deveria tomar, porque os alimentos hoje estão pobres de magnésio. O motivo é simples demais, é que as plantas precisam muito do magnésio para respirar. O mecanismo clorofílico delas - isto é, a fixação do gás carbônico e eliminação do oxigênio - é o contrário do que nós fazemos. Na planta quem faz é a clorofila através do magnésio [20]. 
O fósforo (P) é amplamente distribuído na natureza, sendo encontrado em todas as células, o que significa que todas as fontes alimentares (vegetais ou animais) são potenciais fontes de fósforo. $\mathrm{O}$ fósforo tem a função de auxiliar manutenção do $\mathrm{pH}$, no armazenamento temporário de energia provinda do metabolismo de macronutrientes, na forma de ATP, além de ser responsável pela ativação, por meio da fosforilação de diversas cascatas enzimáticas [21].

O Iodo é um micronutriente essencial para o homem e outros animais encontrado no solo em proporções variadas. Existe apenas uma função conhecida para o Iodo no organismo humano: ele é utilizado na síntese dos hormônios tireoidianos. Estes hormônios têm dois importantes papéis: atuam no crescimento físico e neurológico e na manutenção do fluxo normal de energia (metabolismo basal, principalmente na manutenção do calor do corpo), sendo também importantes para o funcionamento de vários órgãos como o coração, fígado, rins e ovários [22].

Os Distúrbios por Deficiência de Iodo (DDI) são fenômenos naturais e permanentes, que estão amplamente distribuídos em várias regiões do mundo. Populações que vivem em áreas deficientes em iodo podem apresentar os distúrbios causados por esta deficiência, como cretinismo em crianças, anomalias congênitas, bem como a manifestação clínica mais visível: o bócio (hipertrofia da glândula tireoide) [22].

O iodo está distribuído amplamente na natureza e está presente nas substâncias orgânicas e inorgânicas em quantidades muito pequenas. O nível de iodo na água reflete o teor de iodo das rochas e solos da região, e conseqüentemente das plantas comestíveis da região. Normalmente, a água não contribui com uma proporção significativa do consumo diário de iodo, já que mais de $90 \%$ deste provém dos alimentos [22].

A concentração de iodo nas plantas depende propriamente das espécies, tipos de solos, aplicação de fertilizantes no solo, e por fim das condições climáticas. Solos que apresentam alto teor de iodo, tendo como exemplo os solos aluviais e argilosos, geralmente produzem plantas ricas em iodo, sendo dependente da forma química da ocorrência de iodo no solo e sua disponibilidade para a planta [22].

Dentre os metais que podem causar danos à saúde humana pode-se destacar o alumínio $(\mathrm{Al})$, chumbo $(\mathrm{Pb})$, cádmio $(\mathrm{Cd})$, mercúrio $(\mathrm{Hg})$, crômio $(\mathrm{Cr})$, manganês $(\mathrm{Mn})$ e níquel $(\mathrm{Ni})$, estes metais podem ser obtidos por diversas fontes antrópicas [23]. Os efeitos tóxicos dos metais sempre foram considerados como eventos de curto prazo, agudos e evidentes, como a anúria e diarréia sanguinolenta, decorrentes da ingestão de mercúrio.

O excesso destes metais como mercúrio $(\mathrm{Hg})$, chumbo $(\mathrm{Pb})$ e alumínio $(\mathrm{Al})$, entre outros, que vão se acumulando no organismo por exposição crônica aos mesmos, podem causar uma enorme variedade de sintomas, podendo ter uma influência direta em alterações do comportamento por diminuição das funções cerebrais, influenciando na produção e utilização dos neurotransmissores e alterando processos metabólicos [23].

O chumbo $(\mathrm{Pb})$ e o mercúrio $(\mathrm{Hg})$ são metais que não existem naturalmente em nenhum organismo. Não desempenham funções nutricionais ou bioquímicas em microorganismos, plantas ou animais. Ou seja, a presença destes metais em organismos vivos e prejudicial em qualquer concentração. O chumbo, o mercúrio 
e o cadmio são os metais pesados que através dos alimentos, incluindo a agua de consumo, mais tem contribuído para os casos de intoxicação prolongada ou crônica [21,23].

Todas as formas de vida são afetadas pela presença de metais dependendo da dose e da forma química. Muitos metais são essenciais para o crescimento de todos os tipos de organismos, desde as bactérias até mesmo o ser humano, mas eles são requeridos em baixas concentrações e podem danificar sistemas biológicos quando em concentrações elevadas [23,24,25,26].

Em relação ao encontrar o mercúrio (Hg) no mesocarpo do babaçu (Orbignya oleifera). O mercúrio encontrado no mesocarpo de babaçu pode ser proveniente de lagos e lixos contaminantes com pilhas e baterias de carro, pois os mercúrios provenientes dos lixões acabam ocasionando a contaminação da palmeira do babaçu. O coco do babaçu constitui um habitat para inúmeras espécies, sendo que muitas dessas espécies absorvem esse mercúrio. Algumas dessas espécies são consumidas pelo ser humano ocorrendo, assim, a exposição do homem por esta via, pois o mercúrio e facilmente absorvido pelo nosso organismo.

Na figura 2 e tabela 1, pode-se observar o espectro na região do infravermelho do mesocarpo do babaçu. A banda de absorção que aparece na região $3374 \mathrm{~cm}^{-1}$ refere-se à vibração do estiramento da ligação O-H. O alargamento dessa banda deve-se ao fato do grupo hidroxila não estar totalmente livre e participando das ligações de hidrogênio intermoleculares. A absorção em torno da região $2353 \mathrm{~cm}^{-1}$ com intensidade média referem-se às vibrações de estiramentos assimétricos do grupo $\mathrm{CH}_{2}$ e simétricos do $\mathrm{CH}_{3}$ da cadeia longa dos ácidos graxos presentes no babaçu. Na região de absorção $1635 \mathrm{~cm}^{-1}$ aparece o pico com intensidade muito fraca devido à deformação axial do grupo carbonila $\mathrm{C}=\mathrm{O}$. A banda de absorção da ligação carbono-oxigênio, C-O, dos ésteres que aparece na amostra de mesocarpo de babaçu na região de 1149 e 995

$\mathrm{cm}^{-1}$ tem intensidade fraca e muito forte, respectivamente, devido às vibrações de estiramentos assimétricos acoplados à ligação C-O dos ésteres de cadeia longa. A banda na região $856 \mathrm{~cm}^{-1} \mathrm{com}$ fraca intensidade foi atribuída à deformação angular assimétrica no plano $\mathrm{CH}_{2}$ onde todos os grupos metilenos se deformam nesta região.

\section{CONCLUSÃO}

Através deste estudo a espécie Orbignya oleifera, vulgarmente chamada de mesocarpo, pó de babaçu. Foi possível detectar pela análise de metais os macronutrientes (Potássio, Cálcio e Magnésio). Na analise de metais também encontramos uma variedade de metais pesados que possam trazer danos ao ser humano sendo o mercúrio e chumbo em teor significativo. Contudo novos estudos devem ser realizados, para melhor esclarecimento e caracterização sobre a constituição do perfil químico do mesocarpo de babaçu a fim de contribuir de forma ativa ao interesse da sociedade em geral.

\section{AGRADECIMENTOS}

Agradeço ao Instituto Florence de Ensino Superior, Laboratório de solo Universidade Estadual o Maranhão e ao Núcleo de Biocombustíveis, Catalise e Ambiental da Universidade Federal do Maranhão. 


\section{REFERÊNCIAS}

[1] Barbosa, Viviane de Oliveira. Na terra das palmeiras: gênero, trabalho e identidades no universo das quebradeiras de coco babaçu no maranhão / por Viviane de Oliveira Barbosa. - 2007. 160 f.

[2] Lorenzi, H. (org.). Palmeiras brasileiras e exóticas cultivadas. Nova odessa, sp: instituto plantarum, 2004.

[3] Babaçu, uma riqueza maranhense. Bibliografia. Revista de Geografia e História. São Luís: Diretório Regional de Geografia; Conselho Nacional de Geografia; Instituto Brasileiro de Geografia e Estatística, 1961.

[4] Brasil. Ministério do desenvolvimento agrário, ministério do desenvolvimento social e combate a fome \& ministério do meio ambiente. Promoção nacional da cadeia de valor do coco babaçu. Brasília, 2009.

[5] Cruz, E. T. L. Caracterização do Mesocarpo de Babaçu (Orbignya sp.): Termo análise (TG/DTA) e Avaliação do conteúdo de Amido. Monografia do curso de Farmácia. Universidade Federal do Piauí, Teresina, 2011.

[6] Assema. Minha terra tem palmeiras e gente de muita fibra. Pedreiras: assema, 2001.

[7] Jesus, M. M. Estudo do Mesocarpo do Coco Babaçu. São Luís, 1988. 51 pág. Monografia (Química de Alimentos) - Universidade Federal do Maranhão, São Luís - MA, 1988.

[8] Peixoto, Arioso Rodrigues. Plantas oleaginosas arbóreas. Biblioteca rural. Ed. S.A.. São Paulo, 1973.

[9] Silva, Antônio Joaquim da extrativismo do coco babaçu (orbignya phalerata, mart.) No município de Miguel Alves-Pi: caminhos para o desenvolvimento local sustentável / Antônio Joaquim da silva.- 2011. $147 f$.

[10] Wilhelms, C. Babaçu riqueza inexplorada: possibilidades para a intensificação das exportações brasileiras através da diversificação das ofertas. Hamburgo, Alemanha; Rio de Janeiro: Banco do Brasil/Instituto de Óleos, 1968.

[11] Migliato KF, Moreira RRD, Mello JCP, Sacramento LVS, Correa MA, Salgado HRN. Controle de qualidade do fruto de Orbignya Phalerata Mart. Revista Brasileira Farmacognosia. 2007; 17(1): 94-101.

[12] Silverstein, R. M.; Webster, F. X. Identificação espectrométrica de compostos orgânicos. 6. Ed. Rio de Janeiro: Editora Livros Técnicos e Científicos, 2000.

[13] Gobbo-neto, I.; Lopes, N.P. plantas medicinais: fatores de influência no conteúdo de metabólitos secundários. Química nova, v.30, n.2, p.374-81, 2007.

[14] Vidal; Vidal. Disposição dos ramos florais e das flores sobre eles, 1999, p. 29.

[15] Zeitouni, C. De F.; Berton, r. S.; abreu, c. A. De. Fitoextração de cádmio e zinco de um latossolo vermelho-amarelo contaminado com metais pesados. Bragantia, campinas, v. 66, n. 4, p. 649-657, 2007. 
[16] Peixer, Lubna Chagas. Determinação de metais alcalinos e metais alcalinos terrosos em alimentos. Monografia do Curso de Licenciatura em Ciências da Natureza. Instituto Federal de Educação, Ciência e Tecnologia de Santa Catarina - CAMPUS SÃO JOSÉ, 2013.

[17] Peixoto, E. M. A. Elemento químico - potássio. Química nova na escola. № 19 maio 2004.

[18] Catani R. A; paiva neto j. E de. Dosagem do potássio e sódio pelo "fotômetro de chama" - sua aplicação em análise de solo. Trabalho apresentado na II Reunião Brasileira de Ciência do Solo, realizada no Instituto Agronômico de Campinas, de 11 a 22 de julho de 1949.

[19] Nascimento, C. W. A.; xing, b. Phytoextration: a review on enhanced metal availability and plant accumulation. Scientia agricola, v. 63, p. 299-311, 2006.

[20] Schorr, Padre Beno José. O Magnésio Mágico. Para quatro males incuráveis e a maioria dos outros. Adaptado do “Artigo Mirim” - 14 edição, 1994.

[21] Oliveira, Tatiana Coura. Fósforo: função, metabolismo e recomendações. Revista digital de nutrição Ipatinga: Unileste-Mg, v. 1 - n. 1 - ago./dez. 2007.

[22] Vanessa da Matta dos Santos e Júlio Carlos Afonso - Iodo. Química nova escola. Vol.35, № 1 , Fevereiro 2013.

[23] Wood, R. J., Zheng, J. J. High dietary calcium intakes reduce zinc absorption and balance in humans. Am. J. Clin. Nutr., v. 65, n. 1803 1809, 1997.

[24] Rocha, Adriano Ferreira. "cádmio, chumbo, mercúrio - a problemática destes metais pesados na saúde pública?”. Monografia do curso de ciências da nutrição da universidade do porto 2009.

[25] Avila-Campos, Mário Júlio; metais pesados e seus efeitos; disponível: www.mundodoquimico.hpg.com.br: acessado em 11/12/2013.

[26] Fonseca, M. R. M. Da. Química geral. Ftd. São Paulo, 1992. 
Tabela 1: Absorção dos espectros do mesocarpo de babaçu Orbignya oleifera

\begin{tabular}{c|c|c}
\hline Número de ondas $\left(\mathbf{c m}^{-1}\right)$ & Intensidade & Atribuição \\
\hline 3374 & Fraca & $\mathrm{O}-\mathrm{H}$ \\
\hline 2353 & Média & $\mathrm{CH}_{2}, \mathrm{CH}_{3}$ \\
\hline 1635 & Muito fraca & $\mathrm{C}=\mathrm{O}$ \\
\hline 1149 & Fraca & $\mathrm{C}-\mathrm{O}$ \\
\hline 995 & Muito forte & $\mathrm{C}-\mathrm{O}$ \\
\hline 856 & Muito Fraca & $\mathrm{CH}$ \\
\hline
\end{tabular}

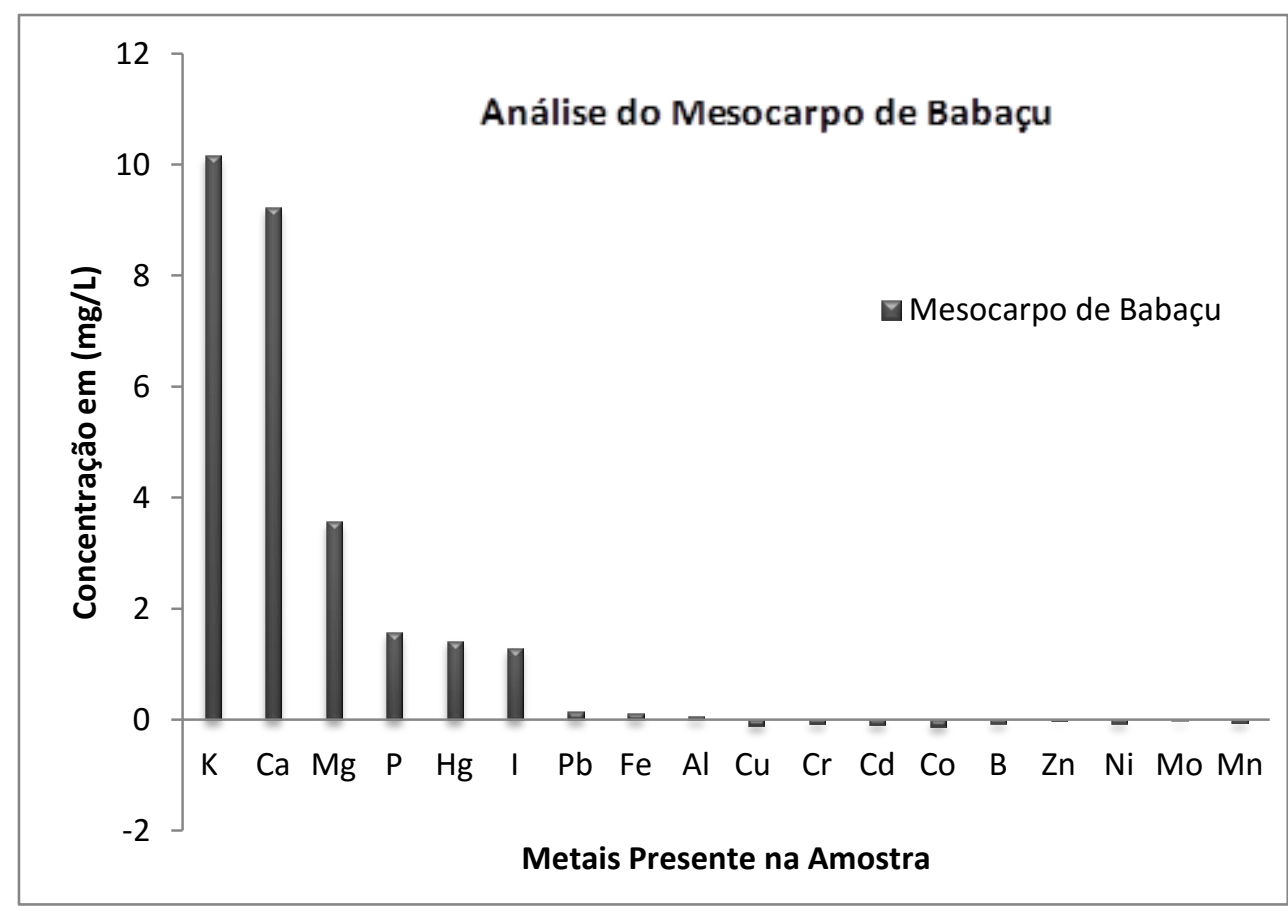

Figura 1 - Análise dos metais pelo método espectrofotométrico (ICP OES) no mesocarpo de babaçu (Orbignya oleífera). 


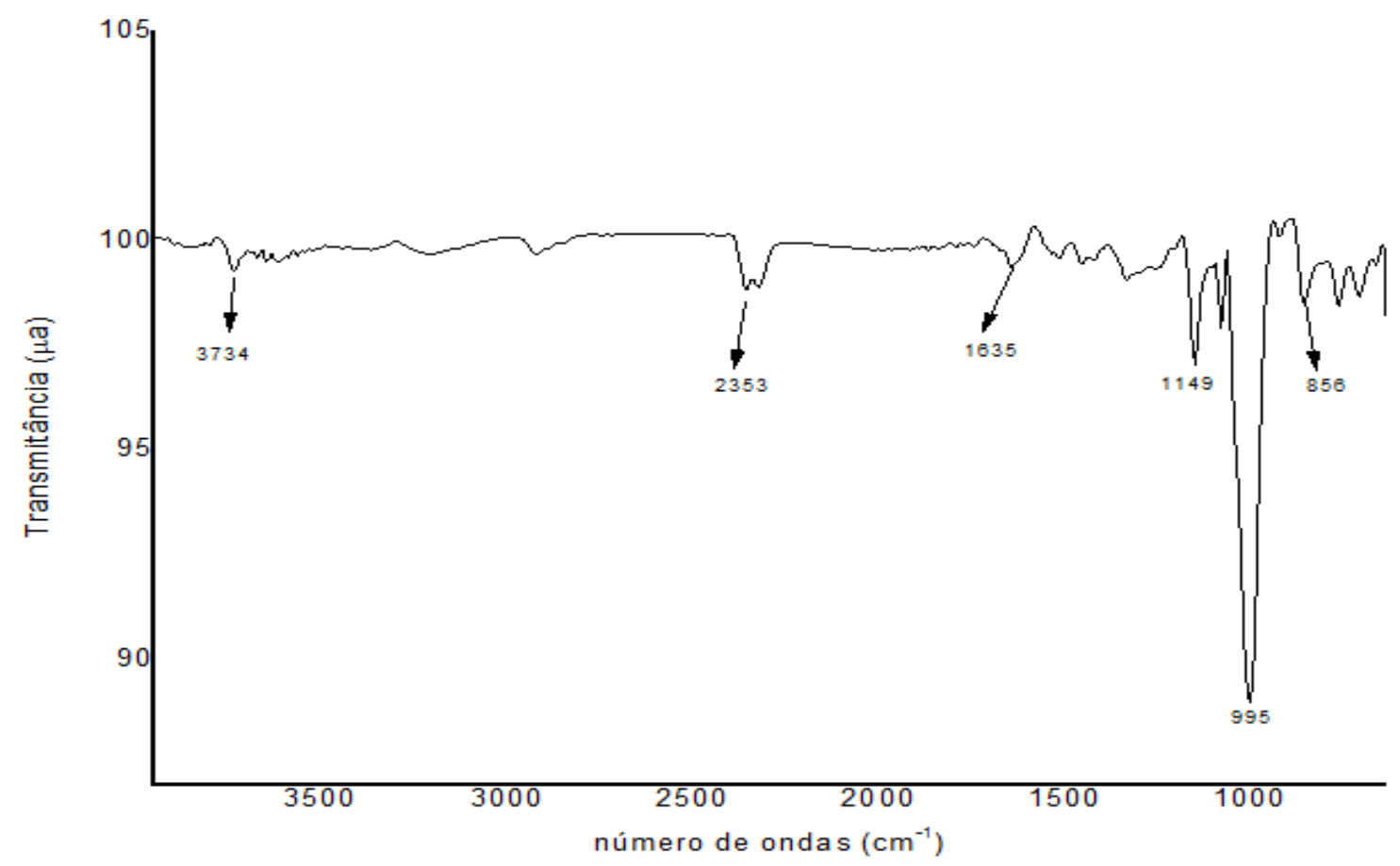

Figura 2: Espectro na região do infravermelho do mesocarpo de babaçu (Orbignya oleifera). 